\title{
Case Report \\ Transvaginal Hybrid NOTES Procedure for Treatment of Gallstone Ileus
}

\author{
Takuya Shiraishi, Naoki Tomizawa, Tatsumasa Andoh, Kazuhisa Arakawa, \\ Yasuaki Enokida, and Naoya Ozawa
}

Department of Surgery, Japanese Red Cross Maebashi Hospital, 3-21-36 Asahi-cho, Maebashi, Gunma 371-0014, Japan

Correspondence should be addressed to Takuya Shiraishi; m04037ts@jichi.ac.jp

Received 19 February 2016; Accepted 9 June 2016

Academic Editor: Cheng-Yu Long

Copyright ( 2016 Takuya Shiraishi et al. This is an open access article distributed under the Creative Commons Attribution License, which permits unrestricted use, distribution, and reproduction in any medium, provided the original work is properly cited.

\begin{abstract}
Gallstone ileus is a rare mechanical bowel obstruction, and previously reported cases have been treated laparoscopically with good results. Although transvaginal hybrid NOTES without a minilaparotomy has been reported to decrease the incidence of surgical wound complications, to our knowledge, this procedure has not been used previously to treat gallstone ileus. We present a case of a 63-year-old woman who underwent transvaginal hybrid NOTES procedure for treatment of gallstone ileus. This case was admitted to our hospital following acute-onset abdominal pain and vomiting. We diagnosed gallstone ileus with cholecystoduodenal fistula by computed tomography and performed totally laparoscopic surgery using only three $5 \mathrm{~mm}$ abdominal ports with transvaginal specimen extraction and enterectomy. The patient's postoperative course was uneventful, and laparoscopic cholecystectomy and fistula repair were performed 8 months after the initial surgery. The patient experienced additional pain relief and good cosmetic outcomes. In conclusion, using transvaginal hybrid NOTES may become a future option to minimize the invasiveness of other laparoscopic procedures.
\end{abstract}

\section{Introduction}

Gallstone ileus is a rare bowel obstruction accounting for $1 \%-3 \%$ of all cases of mechanical obstruction of the small bowel [1]. Gallstone ileus is an abdominal emergency, usually treated surgically. Open enterolithotomy has been the surgery of choice, but many cases are now operated laparoscopically with good results $[2,3]$. However, conventional laparoscopic surgery for gallstone ileus requires an abdominal minilaparotomy for gallstone extraction. Recently, transvaginal hybrid natural orifice transluminal endoscopic surgery (NOTES), which does not involve a minilaparotomy, has been associated with decreased surgical wound complications $[4,5]$. We present a case of transvaginal hybrid NOTES procedure for treatment of gallstone ileus followed by laparoscopic cholecystectomy and fistula repair, 8 months after the initial operation.

\section{Case Presentation}

A 63-year-old woman with mechanical bowel obstruction was referred to our emergency department. She complained of abdominal pain and vomiting that began that morning, and intermittent abdominal pain in the preceding 2 weeks. There was no history of gallbladder disease. On admission, her body mass index was $24 \mathrm{~kg} / \mathrm{m}^{2}$, she did not have a fever, and her abdomen was distended without signs of peritonitis. Laboratory data indicated an elevated white blood cell count of $34.4 \times 10^{9} / \mathrm{L}$ (range, $4.0-9.0 \times 10^{9} / \mathrm{L}$ ), and C-reactive protein level of $19.24 \mathrm{nmol} / \mathrm{L}$ (range, $0.00-2.86 \mathrm{nmol} / \mathrm{L}$ ). Computed tomography (CT) revealed small bowel obstruction by a partially calcified object in the jejunum (Figure 1(a)). Air-fluid levels in the gallbladder were also detected on CT (Figure 1(b)). A cholecystoduodenal fistula resulting from chronic cholelithiasis and gallstone ileus were suspected.

She underwent conservative therapy with antibiotics, and a nasogastric tube was inserted for bowel decompression; however, her abdominal pain did not improve, despite the use of analgesics. Repeat CT revealed that the bowel dilation had improved slightly but that the gallstone position was unchanged.

The patient and her family consented to the recommended operation and also signed a detailed consent form. 


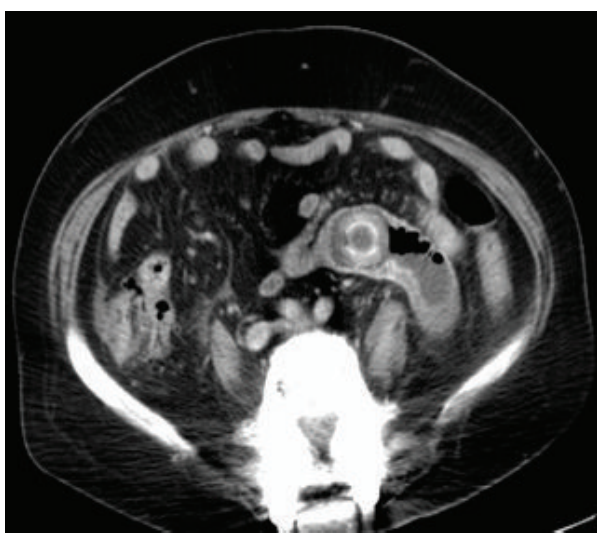

(a)

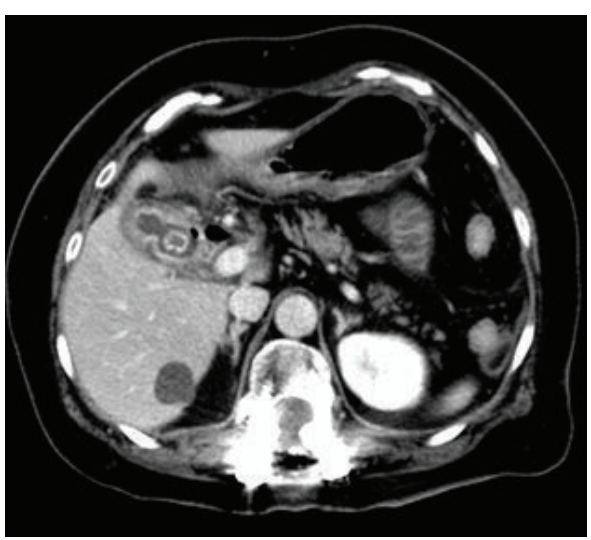

(b)

Figure 1: Computed tomography (CT) images showing (a) small bowel obstruction by a partially calcified object in the jejunum and (b) inflammation around the gallbladder, with a gallstone, and thickening between the gallbladder and the duodenal walls. Air-fluid levels in the gallbladder were also seen.

They also understood the potential risks of the transvaginal approach, and consent was obtained to publish the details of the patient's case.

After induction of general anesthesia, the vagina was washed with $500 \mathrm{~mL}$ of $0.02 \%$ benzalkonium chloride solution. A $5 \mathrm{~mm}$ port was inserted at the navel visually, and pneumoperitoneum was established. Two $5 \mathrm{~mm}$ ports for the laparoscopic instruments were inserted into the upper abdomen, and laparoscopic exploration confirmed the bowel obstruction approximately $70 \mathrm{~cm}$ from the ligament of Treitz. The bowel was thinning at the obstruction, and we were concerned about perforation, postoperatively; therefore, we decided to perform enterectomy with gallstone extraction. After placing the patient in the lithotomy position, a posterior colpotomy was performed using an ultrasonic scalpel under transvaginal guidance. An Alexis bundle wound protector with cap (Alexis Laparoscopic System with Kii Fios First Entry $^{\circledR}$; Applied Medical, Rancho Santa Margarita, CA, USA) was carefully inserted into the abdominal cavity, transvaginally, through the posterior colpotomy. The white ring of the system was covered with the cap that was inserted into a $12 \mathrm{~mm}$ port, maintaining pneumoperitoneum. A liner stapler was then inserted into the abdominal cavity via the vaginal port, and the proximal and distal jejunum at the site of the obstruction were transected (Figure 2). Next, a grasping forceps was inserted into the abdominal cavity, transvaginally, to grasp and remove the specimen with the gallstone. A liner stapler was again inserted into the abdominal cavity, and functional end-to-end anastomosis was performed. The posterior colpotomy incision was closed transvaginally. The laparoscopic surgery for gallstone ileus was completed using three $5 \mathrm{~mm}$ abdominal ports (Figure 3), and the size of the extracted gallstone was $3.5 \times 3.0 \times 3.0 \mathrm{~cm}$ (Figure 4 ). The surgical duration was $162 \mathrm{~min}$, with minimal blood loss. The patient was allowed to drink fluids on postoperative day 2 , and no analgesics were administrated after postoperative day 2. She recovered uneventfully and was discharged on

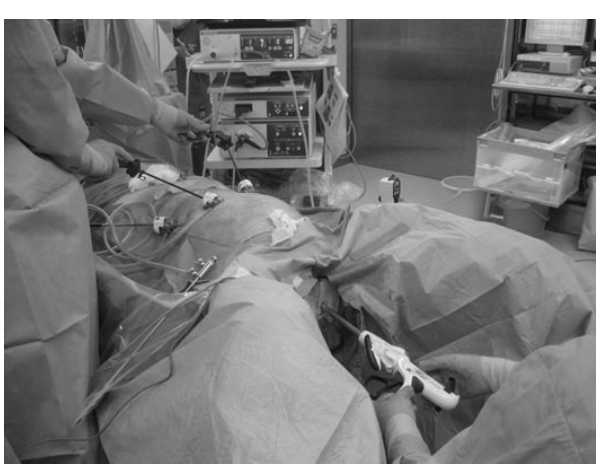

FIGURE 2: Intraoperative image showing the liner stapler being inserted into the abdominal cavity via the vaginal $12 \mathrm{~mm}$ port inserted into the cap.

postoperative day 11. Eight months postoperatively, although she had experienced no recurrence of gallstone ileus and cholecystitis, laparoscopic cholecystectomy and fistula repair were performed. Adhesions were seen around the gallbladder, but there were almost no adhesions at the port sites from first operation. She had an uneventful postoperative course after the second operation and was discharged on postoperative day 5 .

\section{Discussion}

The ideal surgical procedure for gallstone ileus remains controversial $[6,7]$. Reported procedures include enterolithotomy alone, or with the addition of cholecystectomy and fistula repair. Treatment can be performed as a one-stage or two-stage procedure. One-stage surgery has been recommended to reduce the risk of recurrence of gallstone ileus postoperatively, to prevent cholecystitis, and because of the risk of gallbladder malignancy developing in the presence 


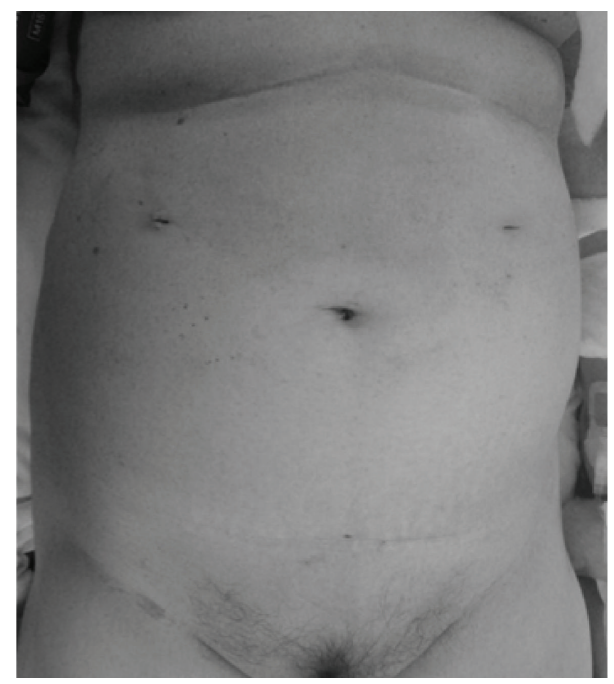

Figure 3: Photograph showing the postoperative scars for the three $5 \mathrm{~mm}$ ports that were inserted into the abdomen.

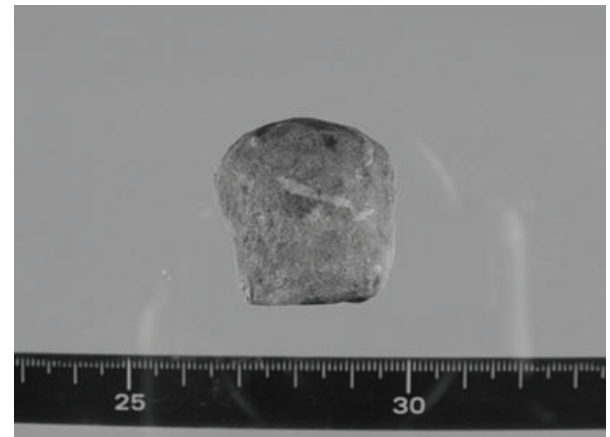

FIgURE 4: The extracted gallstone measuring $3.5 \times 3.0 \times 3.0 \mathrm{~cm}$.

of a fistula [2]. However, one-stage surgery is associated with high mortality and morbidity. Recent studies have reported that enterolithotomy alone is associated with better outcomes $[2,3,6]$, and laparoscopic surgery for gallstone ileus without cholecystectomy and fistula repair has resulted in fewer major complications and shorter hospital stay $[2,3]$. Cholecystectomy and fistula repair are suggested in select patients $[3,6]$.

The transvaginal approach, especially for specimen extraction, is now increasingly performed to reduce the morbidity associated with abdominal wall destruction. This approach is considered safe, because it is established in gynecology $[8,9]$. A meta-analysis reported that transvaginal hybrid NOTES has the advantages of less postoperative pain, low hernia risk, and faster recovery [10]. To our knowledge, ours is the first report to apply transvaginal hybrid NOTES to surgery for gallstone ileus. The diameter of our patient's gallstone was $3.5 \mathrm{~mm}$, which would have required an abdominal incision greater than $3.5 \mathrm{~mm}$ for a conventional laparoscopic approach to extract the specimen and perform enterectomy. We elected to perform not only specimen extraction but also enterectomy via the vaginal route and obtained good results. A $12 \mathrm{~mm}$ port was created in the vagina, and enterectomy was performed using a linear stapler via the vaginal port. We accomplished laparoscopic surgery for gallstone ileus using only three $5 \mathrm{~mm}$ abdominal ports, relieving the patient's pain and obtaining good cosmetic outcomes.

Although transvaginal hybrid NOTES has certain advantages, there are also limitations. First, this procedure demands more time and skill than conventional laparoscopic surgery. Creating the vaginal route takes time, and totally laparoscopic enterectomy demands skills in intracorporeal anastomosis. Second, this procedure cannot be performed for every patient. If surgeons apply this procedure and encounter difficulty, a conventional approach should be adopted to secure patient safety. We believe that these limitations could be resolved with increased skill and improved endosurgical instruments. Further prospective studies are necessary to establish the indications for this procedure, but transvaginal hybrid NOTES may become an option to minimize invasiveness in other laparoscopic surgeries, in the future.

\section{Competing Interests}

The authors declare that there is no conflict of interests regarding the publication of this paper.

\section{References}

[1] Y. Masannat, Y. Masannat, and A. Shatnawei, "Gallstone ileus: a review," Mount Sinai Journal of Medicine, vol. 73, no. 8, pp. 11321134, 2006.

[2] D. J. Soto, S. J. Evan, and M. S. Kavic, "Laparoscopic management of gallstone ileus," Journal of the Society of Laparoendoscopic Surgeons, vol. 5, no. 3, pp. 279-285, 2001.

[3] H. Y. Bircan, B. Koc, U. Ozcelik, O. Kemik, and A. Demirag, "Laparoscopic treatment of gallstone ileus," Clinical Medicine Insights: Case Reports, vol. 7, pp. 75-77, 2014.

[4] C. Palanivelu, M. Rangarajan, P. A. Jategaonkar, and N. V. Anand, "An innovative technique for colorectal specimen retrieval: a new era of 'natural orifice specimen extraction' (N.O.S.E)," Diseases of the Colon and Rectum, vol. 51, no. 7, pp. 1120-1124, 2008.

[5] S. McKenzie, J.-H. Baek, M. Wakabayashi, J. Garcia-Aguilar, and A. Pigazzi, "Totally laparoscopic right colectomy with transvaginal specimen extraction: the authors' initial institutional experience," Surgical Endoscopy and Other Interventional Techniques, vol. 24, no. 8, pp. 2048-2052, 2010.

[6] Y. Watanabe, J. Takemoto, E. Miyatake et al., "Single-incision laparoscopic surgery for gallstone ileus: an alternative surgical procedure," International Journal of Surgery Case Reports, vol. 5, no. 7, pp. 365-369, 2014.

[7] R. A. Gupta, C. R. Shah, and K. P. Balsara, "Laparoscopicassisted enterolithotomy for gallstone ileus," Indian Journal of Surgery, vol. 75, supplement 1, pp. 497-499, 2013.

[8] F. Ghezzi, L. Raio, M. D. Mueller, T. Gyr, M. Buttarelli, and M. Franchi, "Vaginal extraction of pelvic masses following operative laparoscopy," Surgical Endoscopy and Other Interventional Techniques, vol. 16, no. 12, pp. 1691-1696, 2002. 
[9] M. Diana, S. Perretta, J. Wall et al., "Transvaginal specimen extraction in colorectal surgery: current state of the art," Colorectal Disease, vol. 13, no. 6, pp. e104-e111, 2011.

[10] M. H. Sodergren, S. Markar, P. H. Pucher, I. A. Badran, L. R. Jiao, and A. Darzi, "Safety of transvaginal hybrid NOTES cholecystectomy: a systematic review and meta-analysis," Surgical Endoscopy, vol. 29, no. 8, pp. 2077-2090, 2015. 


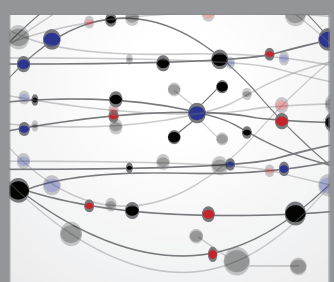

The Scientific World Journal
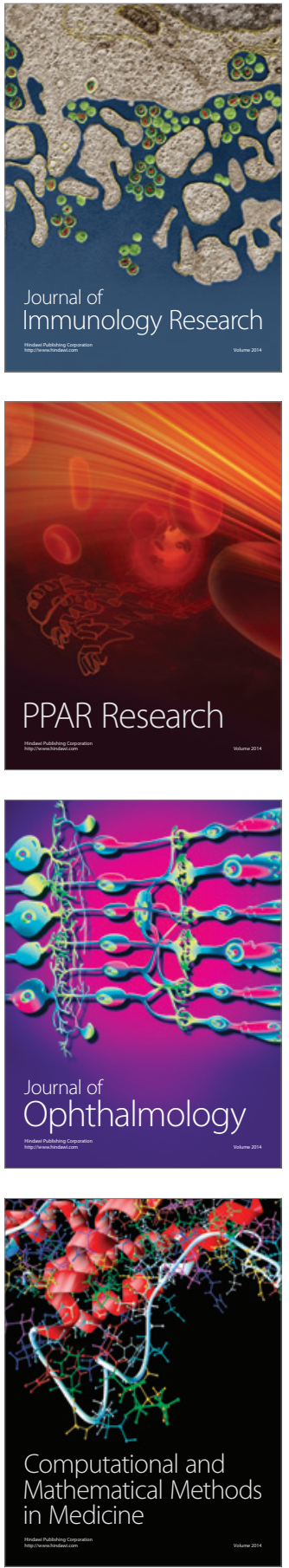

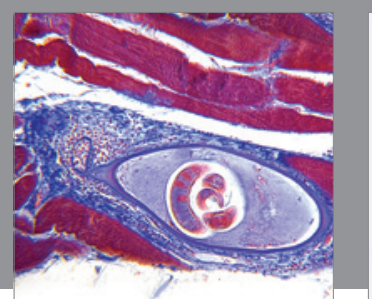

Gastroenterology Research and Practice

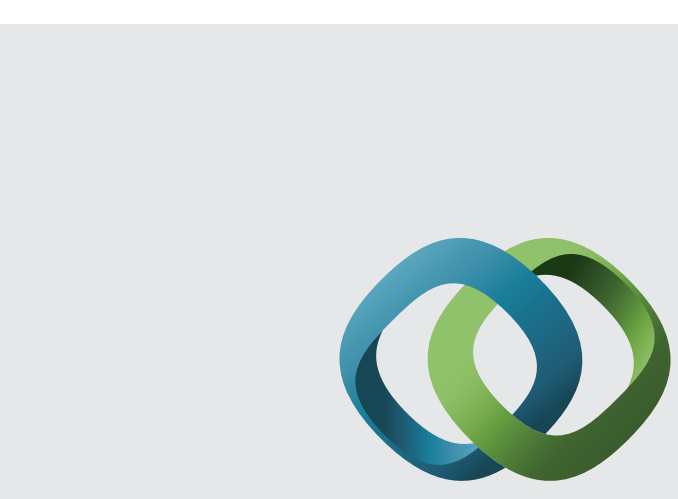

\section{Hindawi}

Submit your manuscripts at

http://www.hindawi.com
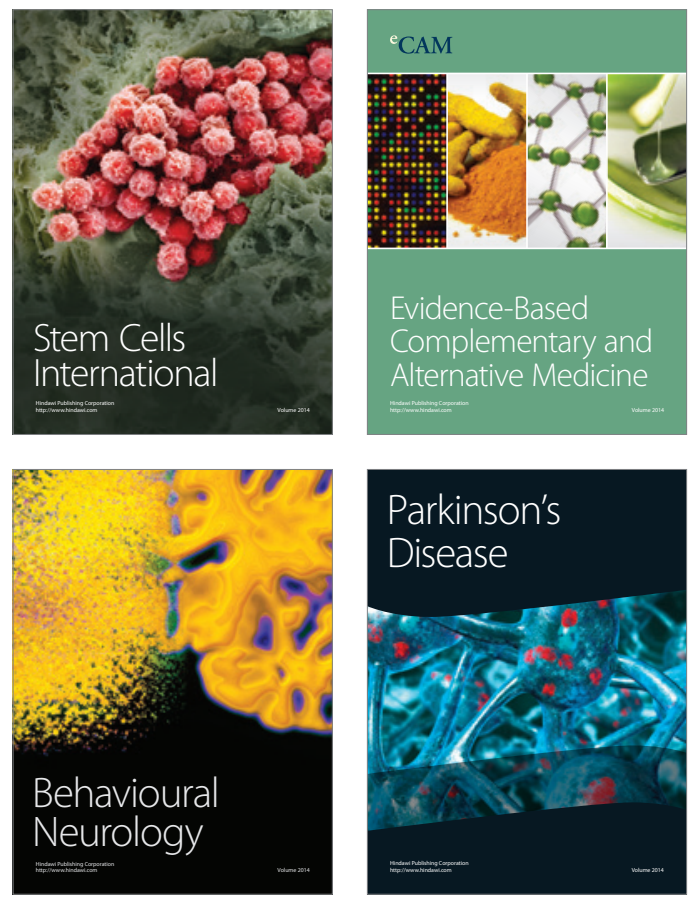
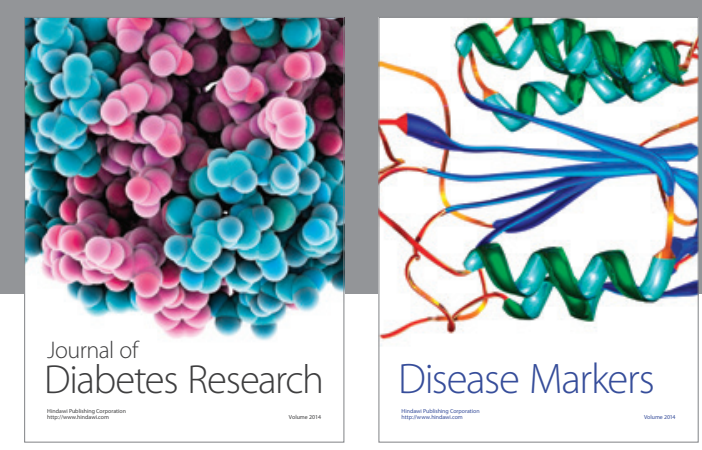

Disease Markers
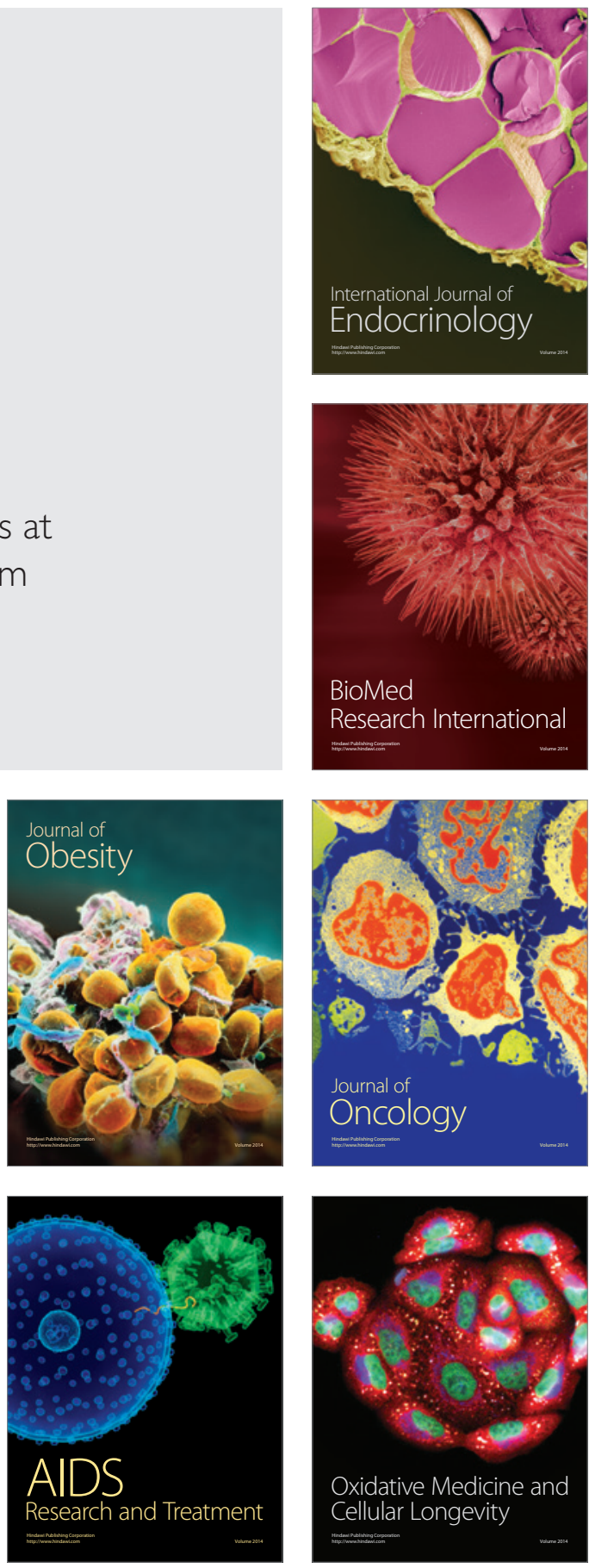